\title{
WT1 as a substrate of HtrA2: a potential pathway for therapeutic targeting by HtrA proteases
}

\section{Jeremy Chien}

Mayo Clinic, Division of Experimental Pathology, Rochester, MN 55905, USA a Tel. : +1 5072844138 n Fax: +1 5072841678 m chien.jeremy@mayo.edu

Evaluation of: Hartkamp J, Carpenter B, Roberts SGE: The Wilms' tumor suppressor protein wt1 is processed by the serine protease HtrA2/Omi. Mol. Cell 37, 159-171 (2010). WT1 is a transcription factor first cloned in 1990 as a tumor suppressor, and inactivating deletions and mutations in WTl are found in childhood kidney Wilms' tumor. The tumor suppressive role of WT1 is further supported by in vitro and in vivo studies in which ectopic expression of WTI attenuated cell growth and tumor formation by promoting apoptosis through induction of proapoptotic proteins. However, WTI is also reported to be overexpressed in adult cancer, and the functional significance of overexpression or de novo expression of WT in adult cancers is not clear, although WT1 in adult cancer may represent isoform-specific differences in WT1 function. A paper by Hartkamp et al. is discussed in this article, reporting the identification of Wilms' tumor suppressor protein WT1 as a substrate of serine protease HtrA2 and the contribution of potential mechanistic insights into how distinct WT1 functions may be regulated through proteolysis by HtrA2.

\section{Summary of methods \& results}

While searching for proteins that bind to the suppression domain of WT1, Hartkamp et al. identified High-temperature requirement A2 $(\mathrm{HtrA} 2)$ as a WT1-interacting protein in a yeast two-hybrid screen using a HeLa cDNA library [1]. A simultaneous screen with a different region of WT1 did not identify HtrA2, indicating the specificity of interaction between WT1 suppression domain and HtrA2. It is important to note that there are four mammalian HtrA proteases, and HtrA2, compared with other $\mathrm{HtrA}$ proteases, is abundantly expressed in $\mathrm{HeLa}$ cells. Therefore, the use of HeLa cDNA library may bias in favor of HtrA2 in this approach. Since HtrA2 is a protease, they then tested the possibility that WT1 may serve as a substrate of HtrA2. In vitro and intracellular assays confirmed that WT1 is an endogenous substrate of HtrA2. In particular, treatment of cells with the chemotherapeutic agent etoposide resulted in the proteolysis of WT1. This proteolysis is in part mediated by HtrA2 because inhibition of HtrA 2 protease activity by UCF-101, a selective inhibitor of serine protease HtrA2, attenuated WT1 proteolysis upon etoposide treatment. UCF-101 is reported to induce cellular responses independent of HtrA2 [2], Hartkamp et al. further demonstrated the specificity of HtrA2 in WT1 proteolysis using siRNAs targeted to HtrA2 and HtrA2 null mouse embryonic fibroblast (MEF) cell models. Hartkamp et al. also demonstrated the presence of $\mathrm{HtrA} 2$ in the nucleus. Since WT1 is a nuclear transcription factor, nuclear localization of HtrA2 further strengthens the possibility that nuclear WT1 may serve as a substrate of nuclear HtrA2. To further define the functional significance of WT1 proteolysis by HtrA2 during apoptosis, Hartkamp et al. downregulated WT1 expression by siRNA and demonstrated that WT1 downregulation promoted apoptosis. Therefore, WT1 acts as an antiapoptotic factor whereas HtrA2 acts as an apoptotic factor. Hartkamp et al. further showed that WT1 downregulation relieves transcriptional repression on c-Myc and JunB, leading to upregulation of these transcripts.

\section{Discussion}

The post-translational modification of protein is a molecular mechanism by which intracellular signals and biological functions are regulated. Proteolysis is one such post-translational modification of proteins, and it is involved in regulating diverse biological functions, including apoptosis, hemostasis and organ homeostasis. Programmed cell death (PCD) is a prime example of biological pathways in which proteolysis controls biochemical pathway that results in cell death. It is also a mechanism by which chemotherapeutic agents exert the desired clinical effect of tumor cell cytotoxicity. Apoptosis, a well-defined morphologic manifestation of PCD, is morphologically characterized by membrane blebbing and nuclear condensation and biochemically characterized by caspases [3] .
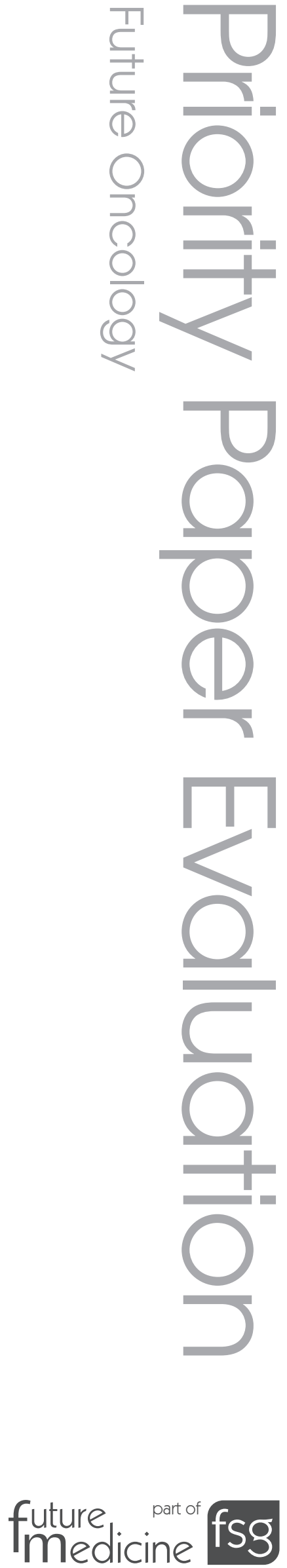
In general, apoptotic signaling cascade is controlled by a fine balance between antiapoptotic signals and apoptotic signals mediated by survival factors (e.g., IAPs, BCL-2, and mediators of growth factor and NFKB signalings) and apoptotic factors (caspases, death receptors and $\mathrm{BH} 3$-only proteins, e.g., BAX, BAK, BID and $\mathrm{BAD}$ ) [4]. Once the balance is tipped in favor of apoptosis subsequent to cytotoxic assault by chemotherapeutic agents, cysteine-aspartic proteases (caspases) act as primary proteolytic enzymes in the apoptotic cascade. However, emerging evidence indicates the regulatory role of serine proteases in PCD [5]. One such serine protease is $\mathrm{HtrA} 2$, which has been shown to target inhibitors of apoptosis (IAPs) during apoptosis, and thereby enhances caspase activation and caspase-mediated cell death [6]. In this paper by Hartkamp et al., an additional role of HtrA2 in apoptosis was uncovered when WT1 was identified as a substrate of HtrA2 [1].

By demonstrating the proteolysis of WT1 by HtrA2 in cells exposed to cytotoxic agents, Hartkamp et al. identified a novel biological pathway by which WT1 may modulate cellular survival and how such survival mechanism is attenuated by proapoptotic serine protease HtrA2. Alterations in transcriptional response produced by WT1 following its proteolysis is also another interesting aspect of the report, although functional significance of altered transcriptional response of c-Myc and JunB is not clear.

This paper identified an important role in which post-translational modification of WT1 by $\mathrm{HtrA} 2$ regulates cellular response to cytotoxic agents. In this proposed model, WT1 acts as an antiapoptotic protein that counters cytotoxic agent-mediated apoptosis. HtrA2, by degrading WT1, antagonizes the antiapoptotic activity of WT1 and promotes cytotoxic agent-mediated apoptosis. Proteolysis of WT1 by HtrA2 also represents an irreversible post-translational modification often characterized in irreversible biological processes, such as apoptosis.

\section{Limitations}

This paper focuses solely on the serine protease HtrA2, and therefore does not address the possible involvement of other members of HtrA serine proteases in WT1 proteolysis. HtrA2 belongs to a family of four mammalian serine proteases consisting of HtrA1, -A2, -A3 and -A4 [7]. Although the role of $\mathrm{HtrA} 2$ in the proteolysis of WT1 is clearly demonstrated, the potential role of $\mathrm{HtrA} 1,-\mathrm{A} 3$ and $-\mathrm{A} 4$ proteases in WT1 proteolysis is not at all explored. Mixture-based peptide library screenings of consensus motifs targeted by HtrA1 and HtrA2 indicate both proteases have strong preference for aliphatic residues in the unprimed positions and polar residues in prime positions of cleavage sites within substrates $[8,9]$. Therefore, HtrA1 and HtrA2 may share some substrates, and HtrA1 may also target WT1 for proteolysis provided that HtrA1 interacts with WT1. In addition, expression and activity of $\mathrm{HtrA} 1$ and $\mathrm{HtrA} 3$ has been shown to be upregulated and activated by chemotherapeutic agents $[10,11]$. Therefore, it will be important to determine whether HtrA1 and HtrA3 interact with WT1 and regulate its proteolysis. Similarly, it will be important to determine which domain within HtrA2 is required by interaction with WT1.

Another limitation in the study relates to the undefined role of c-Myc and JunB in response to cytotoxic agents. Under nonapoptotic conditions, WT1 is responsible for partial suppression of c-Myc and JunB expression. Under apoptotic conditions, WT1 is degraded by HtrA2, thus relieving the suppression by WT and leading to the upregulation of cMyc and JunB expression. However, the functional significance of $\mathrm{cMyc}$ and JunB upregulation following WT1 degradation by HtrA2 is not explored or characterized. In addition, the authors demonstrated the transcriptional upregulation of c-Myc and JunB following WT1 downregulation, but they did not address whether transcriptional upregulation is accompanied by upregulation at protein levels.

\section{Future perspective}

The possibility that HtrA2-mediated proteolysis of WT1 might directly contribute to the effect of cytotoxic drugs warrants additional investigations into the clinical correlation studies between WT1 and HtrA2 in response to chemotherapy. A positive outcome of these studies may further encourage HtrA2-mediated enhancement of chemotherapy in WT1-dependent tumors. One can envision an approach in which HtrA2 agonists, for example, agents that enhance $\mathrm{HtrA} 2$ activity, may be used in combination with conventional chemotherapy to enhance its effectiveness in WT1-dependent tumors. Since protease activity within any given cell is governed by the balance between protease inhibitors with its client protease or protease activity of upstream activating proteases (in the case of zymogens), one can also envisage an approach in which upstream proteases are activated by specific agents or specific protease 
inhibitors are suppressed by RNAi or allosteric neutralization with competitors. In addition, emerging evidence implicate serine proteases in chemotherapy-induced cytotoxicity. There is a potential for cross-talk between caspases and serine proteases in chemotherapy-induced cytotoxicity. Therefore, renewed focus is needed to elucidate these potential cross-talks between different protease pathways to gain functional insights into biochemical proteolysis that precedes chemotherapy-induced programmed cell death. Since the apoptotic pathway is often dysregulated in cancer cells and these alterations are implicated in multifactorial mechanisms of chemotherapy resistance, it is critical to evaluate alternative pathways by which chemotherapeutic agents can induce cytotoxicity. These alternative pathways may become clinically relevant targets to augment cell death mediated through caspasemediated proteolytic cascades. Therefore, studies reported by Hartkamp et al. open up novel avenues for potential therapeutic interventions in cancer.

Financial \& competing interests disclosure
The author has no relevant affiliations or financial
involvement with any organization or entity with a
financial interest in or financial conflict with the sub-
ject matter or materials discussed in the manuscript.
This includes employment, consultancies, honoraria,
stock ownership or options, expert testimony, grants or
patents received or pending, or royalties.
No writing assistance was utilized in the production
of this manuscript.

\section{Executive summary}

\section{Study design}

- Molecular and functional characterization of the interaction between WT1 and HtrA2.

\section{Methods}

- In vitro labeling, translation and degradation of WT1 by recombinant HtrA2.

- Immunoblot analyses of intracellular WT1 degradation by HtrA2 and cytotoxic agents.

- Modulation of HtrA2 and WT1 expression by RNAi.

- Subcellular localization of HtrA2 by fractionation.

- Chromatin immunoprecipitation.

\section{Results}

- HtrA2 specifically cleaves WT1 in vitro and in cells.

- HtrA2 is required for the degradation of WT1 following exposure to cytotoxic agents.

- Endogenous HtrA2 can be localized in both cytoplasmic and nuclear fractions.

- WT1 is upregulated in HtrA2-knockout mouse embryonic fibroblasts.

- WT1 knockdown by siRNA enhances poly(ADP-ribose) polymerase (PARP) cleavage is induced by the cytotoxic agent Staurosporine.

- WT1 knockdown by siRNA upregulates c-Myc and JunB transcripts.

- WT1 cleavage by HtrA2, following Staurosporine treatment, upregulates c-Myc and JunB transcripts.

\section{Conclusion}

WT1 is processed by the serine protease HtrA2.

\section{Bibliography}

1. Hartkamp J, Carpenter B, Roberts SG: The Wilms' tumor suppressor protein WT1 is processed by the serine protease HtrA2/Omi. Mol. Cell 37(2), 159-171 (2010).

2. Klupsch K, Downward J: The protease inhibitor Ucf-101 induces cellular responses independently of its known target, HtrA2/Omi. Cell Death Differ. 13(12), 2157-2159 (2006).

3. Danial NN, Korsmeyer SJ: Cell death: critical control points. Cell 116(2), 205-219 (2004).

4. Igney FH, Krammer PH: Death and anti-death: tumour resistance to apoptosis. Nat. Rev. Cancer 2(4), 277-288 (2002).
5. Leist M, Jaattela M: Four deaths and a funeral: from caspases to alternative mechanisms. Nat. Rev. Mol. Cell. Biol. 2(8), 589-598 (2001).

6. Suzuki Y, Imai Y, Nakayama H, Takahashi K, Takio K, Takahashi R: A serine protease, $\mathrm{HtrA} 2$, is released from the mitochondria and interacts with XIAP, inducing cell death. Mol. Cell 8(3), 613-621 (2001).

7. Clausen T, Southan C, Ehrmann M: The HtrA family of proteases: implications for protein composition and cell fate. Mol. Cell 10 (3), 443-455 (2002).

8. Martins LM, Turk BE, Cowling V et al: Binding specificity and regulation of the serine protease and PDZ domains of $\mathrm{HtrA} 2 / \mathrm{Omi}$. J. Chem. 278(49), 49417-49427 (2003).
9. Chien J, He X, Shridhar V: Identification of tubulins as substrates of serine protease HtrA1 by mixture-based oriented peptide library screening. J. Cell. Biochem. 107(2), 253-263 (2009).

10. Chien J, Aletti G, Baldi A et al.: Serine protease HtrA1 modulates chemotherapyinduced cytotoxicity. J. Clin. Invest. 116(7), 1994-2004 (2006).

11. Beleford D, Rattan R, Chien J, Shridhar V: High temperature requirement A3 (HtrA3) promotes etoposide- and cisplatin-induced cytotoxicity in lung cancer cell lines. J. Biol Chem. 285(16), 12011-12027 (2010). 